\title{
Estrogen differentially alters the activity of male rats in plain and enhanced environments
}

\author{
CHARLES H. K. WEST and RICHARD P. MICHAEL \\ Emory University School of Medicine, Atlanta, Georgia \\ and Georgia Mental Health Institute, Atlanta, Georgia
}

\begin{abstract}
To enable investigation of the short latency effects of estradiol, locomotor activity was monitored during a single 10 -min session in adult male rats $(n=71)$ that were either naive or adapted to the experimental testing procedures. All animals were administered either oil or $50 \mu \mathrm{g} / \mathrm{kg}$ estradiol benzoate (EB) 30 min before the session and were tested in an environment that was either plain or enhanced with four odorants (ethanol, ammonia, cedar oil, and female rat urine). Compared with activity of control animals, the activity of naive rats was decreased both by EB treatment and by odor enhancement of the environment, but activity was not reduced in naive animals by the combination of EB treatment and odor enhancement. In contrast to the activity of naive animals, the activity of adapted animals was not affected by EB treatment, odor enhancement, or a combination of both. The behavior of naive rats during locomotor activity testing suggested that a short-latency, presumably nongenomic, effect of EB altered the animals' reactions to odors in the novel environment.
\end{abstract}

A number of behaviors not directly related to reproduction are known to be influenced by estrogens and other gonadal hormones (Beatty, 1979). In recent years, several reports have drawn attention to the effects of estrogen on the midbrain dopaminergic systems (Van Hartesveldt \& Joyce, 1986), and the observed effects depend on many factors, including the time interval between estrogen administration and the test of dopamine (DA) function (Gordon, 1980). These studies have examined DA function either behaviorally or biochemically 1 or more days after estrogen administration, a time interval sufficient to allow for activation of the genomic mechanisms of steroid hormone action. However, electrophysiological studies have shown that estrogen can produce neuronal effects with latencies of only a few seconds (Chiodo \& Caggiula, 1983; Haskins \& Moss, 1983) or minutes (Nabekura, Oomura, Minami, Mizuno, \& Fukuda, 1986).

Recent work in our laboratory has demonstrated a shortlatency, presumably nongenomic, effect of estrogen on DA-mediated behavior in male rats (West \& Michael, 1986). In this study, amphetamine-induced hyperactivity was maximally increased $30 \mathrm{~min}$ after a single injection of estradiol benzoate (EB). A confounding factor in this and in other studies employing drugs to evaluate DA function is that estrogen might alter the potency, metabolism, and distribution of the drug in the brain independently of an effect on dopaminergic neurons themselves (Chiodo, Caggiula, \& Saller, 1981). A method of studying estrogen's actions that avoids some of the pharmacodynamic problems involves monitoring behavior that reflects a

General research support was provided by the Georgia Department of Human Resources, which is gratefully acknowledged. Address correspondence to Charles H. K. West, Georgia Mental Health Institute, 1256 Briarcliff Road, N.E., Atlanta, GA 30306. physiologically induced increase in DA function. One such behavior, associated with DA transmission in the mesocorticolimbic system, is the high level of exploratory activity displayed by male rats during their initial exposure to a novel environment (Fink \& Smith, 1980a, 1980b; E. M. Joyce, Stinus, \& Iversen, 1983).

In the present study, we investigated the action of estrogen on the hyperactivity induced by a novel environment to ascertain whether a short-latency estrogen effect can be observed without the administration of any dopaminergic drugs. Because estrogens have been implicated in the sex differences that occur with different sensory inputs (Gandelman, 1983) as well as with novel environments (Archer, 1975), the influence of enhancing the novel environment with odor stimuli was also examined. We reasoned that the presence of any additional sensory stimulus might influence exploratory activity and interact with an estrogen effect if one were observed.

\section{METHOD}

\section{Subjects}

Eighty-seven sexually naive male Sprague-Dawley rats, raised from breeding stock in our Laboratory (Charles River, Wilmington, MA), were used. Seventy-one were used for behavioral testing, and 16 were used for additional corticosterone measurement. To reduce both the influence of endogenous estrogens and the greater variability of locomotor activity in female rats in novel environments (Archer, 1975), male rats were used. It was felt that gonadectomy was best avoided since the design called for naive animals. At the time of the experiment, the rats were 14 weeks old and weighed $375-550 \mathrm{~g}$. They were housed 3-4 per cage in a colony room lighted between 7:00 a.m. and 7:00 p.m., and they had free access to fresh food and water.

\footnotetext{
Apparatus

Locomotor activity was measured by infrared beam interruptions using a Digiscan RXY activity monitor (Omnitech, Columbus, $\mathrm{OH}$ ).
} 
Rats $(n=71)$ were placed in an acrylic box with a covering lid $(406 \times 406 \times 311 \mathrm{~mm})$, and the box was positioned within the activity monitor. The monitor itself was placed inside a soundattenuating chamber equipped with a fan and a $25-\mathrm{W}$ red light bulb. The locomotor activity of each animal was monitored in a single 10-min session between 9:00 a.m. and 12:30 p.m. The box was thoroughly washed, rinsed, and dried between sessions to prevent the retention of odors. On the day of testing, all animals were transported to the testing area approximately $1 \mathrm{~h}$ before the first animal was injected.

\section{Estrogen Treatment}

Thirty minutes before activity testing, each animal received subcutaneous injections of either sesame oil $(0.25 \mathrm{ml} / \mathrm{kg})$ or $17 \beta$ estradiol benzoate (EB; $50 \mu \mathrm{g} / \mathrm{kg}$ ) (Sigma Chemical Co., St. Louis, $\mathrm{MO}$ ). This dose of EB is supraphysiological, but it has been used by others to investigate the effects of estrogen on the midbrain DA system (Van Hartesveldt \& Joyce, 1986), and in previous work by us it was effective in increasing amphetamine-induced hyperactivity (West \& Michael, 1986).

\section{Odor Stimuli}

It should be emphasized that odor stimuli were used here simply to enhance the sensory properties of the environment, and not because of a primary interest in the physiological properties of the odors themselves, which would require a different experimental design. The four odorants were $100 \%$ ethanol, ammonia (33\% ammonium hydroxide diluted 5:1 with distilled water), cedar wood oil, and female rat urine (freshly collected). Pooled urine from 6 intact female rats at different stages of the estrous cycle was collected by syringe from a clean plastic cage uncontaminated by feces or water. These particular odors were used because previous electrophysiological experiments with male rats had shown that they were effective in changing the rate of firing of nucleus accumbens neurons (West \& Michael, 1985). The four odorants were used in combination in these initial tests to enhance the strength of the stimulus and to increase the likelihood of there being at least one effective odorant. To prevent animals from having direct contact with the odor source, $0.5 \mathrm{ml}$ of the odorant was applied to a cotton ball in the bottom of a small test tube $(75 \times 12 \mathrm{~mm})$. Four test tubes, each containing one of the odorants, were placed in the corners of the activity testing box. The position of the tubes was randomized for each animal, and fresh odorants were applied between sessions. For tests in the plain environment, four empty, clean test tubes were placed in the corners of the acrylic box for control purposes (e.g., exploration).

\section{Adaptation and Activity Testing Procedures}

At the time data were collected, rats were either experimentally naive or adapted to the testing procedure. Twenty-four animals were adapted to activity testing and to the injection procedures in 14 daily 10 -min sessions over a period of 3 weeks. During the last 2 of these sessions, the testing environment was odor enhanced. The naive $(n=47)$ and adapted $(n=24)$ animals were randomly divided into eight groups. Ages and weights did not differ significantly among groups. The testing environment was either plain or odor enhanced. The resultant four treatment groups for both naive and adapted animals were oil injected + plain environment $(\mathrm{Oil}+\mathrm{Pl})$, estradiol injected + plain environment $($ Est $+\mathrm{Pl})$, oil injected + odorous environment $(\mathrm{Oil}+\mathrm{Od})$, and estradiol injected + odorous environment $($ Est + Od).

\section{Direct Observations During Activity Testing}

The final 20 of the 47 naive animals used were observed during activity testing, and their corticosterone levels were assayed (see below). The mean locomotor activity scores of the animals that were and were not used for these measurements did not differ significantly for the four treatment groups. The presence or absence of the following eight categories of behavior was noted by the experimenter: maintained exploration (active more than $75 \%$ of the session), brief exploration (active less than $50 \%$ of the session), rearing (repeated), little sniffing of odors (brief sniffing of odorants a few times), much sniffing of odors (repeated and prolonged sniffing of several odorants), grooming, and general inactivity (little or no movement for most of the session). The same experimenter made all injections and behavioral observations. Since these observations were not precisely quantified, the results were not analyzed statistically and are included only to help interpret the locomotor activity data.

\section{Corticosterone Measurement}

Since stress can be induced in naive rats by a novel environment, and this may influence exploratory activity, we wished to assess the level of stress produced by the different experimental conditions. Plasma corticosterone levels were therefore measured in six groups of naive rats. To facilitate comparisons with experimental animals, two separate groups of naive rats that received no activity testing were killed by decapitation either within 1 min after removal from their home cages in the colony room (resting, $n=8$ ) or after 15 min of restraint stress (stressed, $n=8$ ). The latter animals were restrained inside a snug-fitting cylinder, which restricted gross movements but did not cause pain or impair respiration. Some rats $(n=20)$ from each of the four groups of naive experimental animals also were killed by decapitation immediately after activity testing. Trunk blood from all animals was collected and centrifuged, and plasma samples were immediately frozen. Plasma corticosterone levels were measured by radioimmunoassay (Innovet, Cambridge Medical Diagnostics, Billerica, MA) using a double antibody method without extraction on $5 \mu \mathrm{l}$ aliquots of plasma. The intraassay coefficient of variation was $9.8 \%$, and the sensitivity (blank + two standard deviations) was $0.034 \mathrm{ng} / \mathrm{ml}$.

\section{Data Analysis}

The number of beam interruptions per 10-min test provided information on locomotor activity. Analyses of variance followed by Scheffe's test were used to assess the significance of differences between group means (SPSS/PC+, SPSS Inc., Chicago, IL). Due to the small number of subjects for some of the corticosterone assay groups, analysis of variance followed by the less rigorous Duncan multiple-range test was used to assess the data.

\section{RESULTS}

The activity of naive rats in a novel testing environment was changed by estrogen treatment and odor enhancement of the environment (Figure 1, upper part). Analysis of variance confirmed that there was a highly significant effect of the treatment condition on locomotor activity scores $[F(3,43)=17.47, p<.001]$. Activity was significantly reduced in rats receiving EB treatment $(p<.001)$ in a plain environment $($ Est $+\mathrm{Pl})$ compared with oil-treated controls (Oil $+\mathrm{Pl}$ ). Odor enhancement of the environment of oil-treated animals had a very similar effect to that of estrogen treatment, and activity was also significantly reduced $(p<.001)$ in the odor-enhanced environment (Oil + Od) compared with the plain environment (Oil + Pl) (Figure 1, upper part). However, when EB treatment and odor enhancement were combined, there was no change in activity scores compared with control levels. Thus, in the odor-enhanced environment, EB treatment increased activity compared with oil treatment, and the Est + Od group showed a significantly $(p<.001)$ 

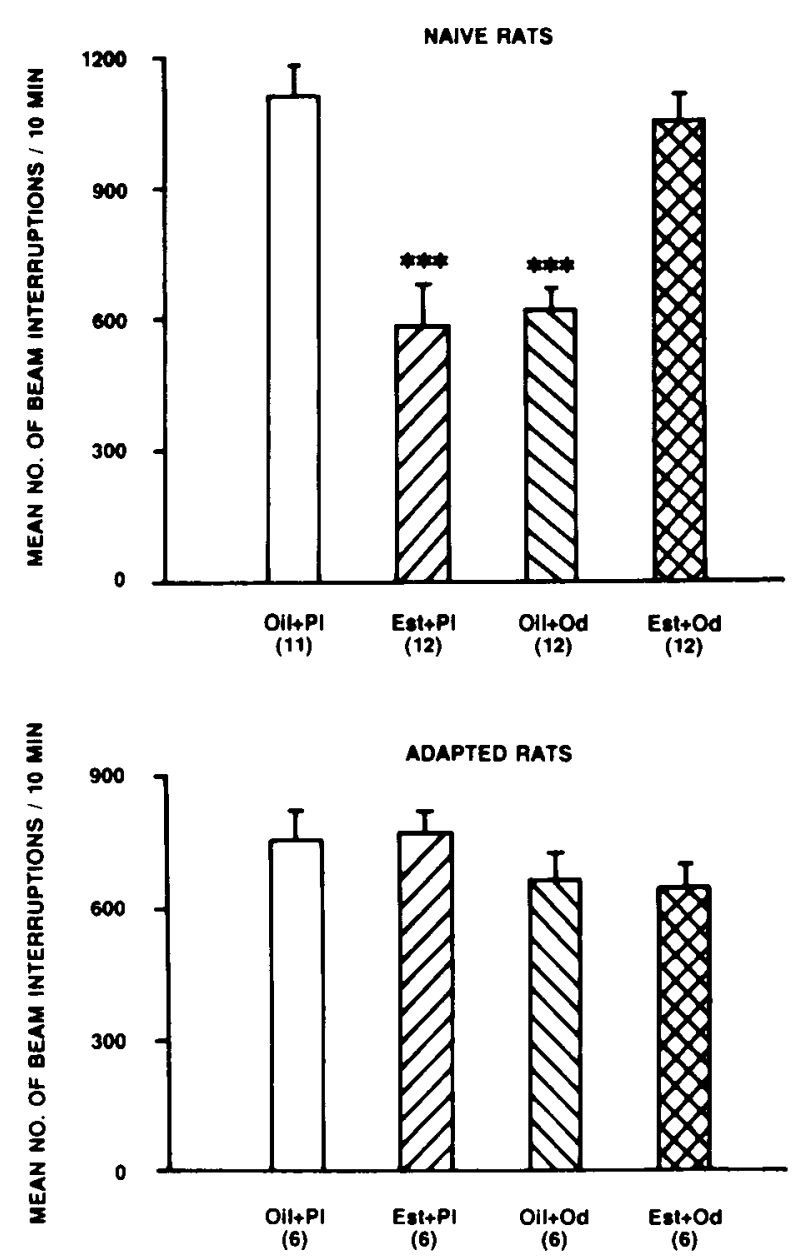

Figure 1. Mean ( $t$ standard error of the mean) locomotor activity scores per 10-min session for four groups of naive male rats in a novel environment (upper part) and for four groups of adapted rats in a familiar environment (lower part). Animals were administered oil or estradiol benzoate $(50 \mu \mathrm{g} / \mathrm{kg}) 30 \mathrm{~min}$ before the session and were tested in a plain or an odor-enhanced environment. The four treatment groups were oil + plain environment $(\mathrm{Oil}+\mathrm{PI})$, estrogen + plain environment (Est + PI), oil + odor-enhanced environment (Oil +Od), and estrogen + odor-enhanced environment (Est +Od). The numbers of animals are given in parentheses. In naive rats, means of the Est $+\mathrm{PI}(586 \pm 87)$ and $\mathrm{O} i 1+\mathrm{Od}(623 \pm 44)$ groups were significantly different from the mean of the control Oil + P1 group $(1,106 \pm 60)$ by analysis of variance. $\quad * * * p<.001$.

higher mean number of beam interruptions $(1,054 \pm 60)$ than the Oil + Od group (623 \pm 44$)$. Figure 1 (lower part) shows that there were no significant differences of any kind in the locomotor activity of adapted animals $[F(3,20)$ $=1.25, p>.1]$.

Table 1 shows the proportion of naive animals engaged in various behaviors during activity testing. Behavior was observed for only some members of each group, but these data facilitate the discussion of mechanisms that may underlie the observed effects on activity. Noteworthy differences between groups occurred in maintained exploration, shown by 4 out of $5 \mathrm{Oil}+\mathrm{Pl}$ animals, but by none of the $4 \mathrm{Oil}+\mathrm{Od}$ and none of the $6 \mathrm{Est}+\mathrm{Pl}$ animals; sniffing air, shown by 3 out of $4 \mathrm{Oil}+\mathrm{Od}$ and 4 out of 5 Est $+\mathrm{Od}$ animals, but by only 1 out of $5 \mathrm{Oil}+\mathrm{Pl}$ and none of the 6 Est $+\mathrm{Pl}$ animals; and much sniffing of odors, shown by all 5 of the Est $+\mathrm{Od}$ animals, but by none of the $4 \mathrm{Oil}+\mathrm{Od}$ animals.

Plasma corticosterone levels differed significantly among the six groups of naive animals, as indicated by analysis of variance $[F(5,30)=3.26, p<.05]$. The mean corticosterone level of the stressed group $(142 \pm 12 \mathrm{ng} / \mathrm{ml})$ was significantly $(p<.05)$ higher than that of the resting group $(57 \pm 4 \mathrm{ng} / \mathrm{ml})$ but was not significantly different from the means of the four activity tested groups (Oil $+\mathrm{Pl}=107 \pm 24 \mathrm{ng} / \mathrm{ml}$, Est $+\mathrm{Pl}=$ $106 \pm 13 \mathrm{ng} / \mathrm{ml}, \mathrm{Oil}+\mathrm{Od}=192 \pm 74 \mathrm{ng} / \mathrm{ml}$, and Est $+\mathrm{Od}$ $=105 \pm 26 \mathrm{ng} / \mathrm{ml}$ ). The mean corticosterone level of the Oil + Od group was somewhat higher than the means for the Est $+\mathrm{Pl}$ and resting groups. Although the Oil $+\mathrm{Od}$ group showed the highest mean corticosterone level, one animal in this group had a very high value $(403 \mathrm{ng} / \mathrm{ml})$, which distorted the results and prevented any conclusions from being drawn.

\section{DISCUSSION}

The results demonstrated that a short-latency $(30 \mathrm{~min})$ effect of estrogen reduced the exploratory activity of naive male rats in a novel environment. Since it has been shown that normal investigatory activity in novel environments requires DA transmission in the mesocorticolimbic system (Fink \& Smith, 1980a), one might speculate that the effect was due to a short-latency estrogenic modulation of DA function. The fact that the mesocorticolimbic DA system is involved with exploratory activity only in a novel environment and not in a familiar environment is quite consistent with this view (Fink \& Smith, 1980b). In the present study, the estrogen effect occurred with naive rats in a novel environment but not with adapted rats in a familiar environment. As anticipated, locomotor activity scores of the Oil + Pl controls were higher during the exploration of a novel environment by the naive animals $(1,109 \pm 66)$ than during the reinvestigation of a familiar environment by the adapted animals $(754 \pm 75)$. EB treatment affected the activity of naive, but not of adapted, animals. This was in line with the previous finding that EB affected hyperactivity induced by amphetamine but not spontaneous activity following saline administration (West \& Michael, 1986), and this suggested that elevated activity levels may be necessary to demonstrate estradiol's short-latency effects.

Previous studies have shown that effects on behavior mediated by the midbrain DA systems appear within a few hours after the last estrogen treatment, but both increases (Becker \& Beer, 1986; Bedard, Boucher, Daigle, \& Di Paolo, 1985; Di Paolo, Rouillard, \& Bedard, 1985; West \& Michael, 1986) and decreases (J. N. Joyce, Montero, \& Van Hartesveldt, 1984; J. N. Joyce \& Van Hartesveldt, 1984) in DA function have been reported. It is possible that estrogen produces differential short-latency 
Table 1

Proportion of Naive Animals Showing Various Behaviors During the Locomotor Activity Session

\begin{tabular}{lcccc}
\hline \multicolumn{1}{c}{ Behaviors } & Oil + Plain & Estradiol + Plain & Oil + Odors & Estradiol + Odors \\
\hline Maintained exploration & $4 / 5$ & $0 / 6$ & $0 / 4$ & $2 / 5$ \\
Brief exploration & $1 / 5$ & $3 / 6$ & $2 / 4$ & $1 / 5$ \\
Rearing & $3 / 5$ & $0 / 6$ & $3 / 4$ & $4 / 5$ \\
Sniffing air & $1 / 5$ & $0 / 6$ & $3 / 4$ & $4 / 5$ \\
Little sniffing of odors & - & - & $2 / 4$ & $0 / 5$ \\
Much sniffing of odors & - & - & $0 / 4$ & $5 / 5$ \\
Grooming & $2 / 5$ & $3 / 6$ & $3 / 4$ & $5 / 5$ \\
General inactivity & $0 / 5$ & $3 / 6$ & $0 / 4$ & $0 / 5$ \\
\hline
\end{tabular}

Note-Behavior of 20 of the 47 naive animals was observed during activity testing

effects on dopaminergic transmission via different pathways and mechanisms, depending on such factors as dose of hormone and use of dopaminergic drugs. For example, estrogen has been shown to enhance the release of DA induced by amphetamine (Becker \& Beer, 1986), and this mechanism may explain the enhancement by EB of amphetamine-induced hyperactivity in male rats. In contrast, when the same dosage and injection-test interval were employed in the present study, EB reduced the novelty-induced hyperactivity, perhaps by means of an antidopaminergic effect at postsynaptic receptors (J. N. Joyce et al., 1984; J. N. Joyce \& Van Hartesveldt, 1984). Alternatively, the effect described here may have involved non-DA systems or a different portion of the midbrain DA pathway (Alderson \& Baum, 1981; Vaughn, Viik, \& Cooper, 1985).

Enhancing a novel environment with odors decreased the activity of naive rats during exploration. One plausible explanation is that animals spent more time sniffing and investigating the odor sources and less time moving about the box, but direct observations indicated that only half of the Oil + Od rats showed little sniffing, and none showed much sniffing (Table 1). Another possibility is that the novelty of the test environment stressed the naive rats and that the addition of sensory stimuli further increased their emotionality, which decreased exploratory activity. We found that, compared with resting levels, plasma corticosterone was elevated following the stress of restraint, and this was consistent with the findings of others (e.g., Kant, Mougey, \& Meyerhoff, 1986). Our naive rats tested in a novel environment also showed elevated corticosterone levels, although generally to a lesser extent than those produced by restraint. It is possible that an animal may tend to avoid potent olfactory stimuli in a stressful situation, such as a novel environment, and show less active exploration because of this. This possibility was supported by the observation that maintained exploration was shown by more of the $\mathrm{Oil}+\mathrm{Pl}$ animals $(80 \%)$ than by the Oil + Od animals $(0 \%)$, whereas sniffing the air was shown by more of the Oil + Od animals (75\%) than by the Oil + Pl animals (20\%) (Table 1). To determine which of the odorants was predominantly responsible for the effect would require their separate assessment in another series of experiments.

The reversal by EB of the decrease in activity produced by odor enhancement surprised us since EB alone also decreased activity. At longer latencies, estrogen has been shown to influence both emotionality and sensory function in rats (Archer, 1975; Gandelman, 1983), making it difficult to identify the precise mode of action in the present situation. However, observing the behavior of naive rats seemed to point to a change in sensory function. For example, more of the Est +Od animals $(100 \%)$ showed much sniffing of odors than did the Oil $+\mathrm{Od}$ animals $(0 \%)$, and it could be that estradiol was inducing a distinct change in the way the animals perceived, and reacted to, the odors. Estradiol might also reduce the reactivity of animals to novel olfactory stimuli. Perhaps estrogen reduced the activity-limiting quality, that is, the perceived intensity or novelty of the odorants. Whether a change in odor perception or an effect of EB on DA function was primarily involved cannot be determined from these data, and we do not know whether estrogen in physiological amounts has any short-latency effects on odor perception. These are questions that warrant further study.

\section{REFERENCES}

Alderson, L. M., \& BAuM, M. J. (1981). Differential effects of gonadal steroids on dopamine metabolism in mesolimbic and nigro-striatal pathways of male rat brain. Brain Research, 218, 189-206.

ARCHER, J. (1975). Rodent sex differences in emotional and related behavior. Behavioral Biology, 14, 451-479.

BEATTY, W. W. (1979). Gonadal hormones and sex differences in nonreproductive behaviors in rodents: Organizational and activational influences. Hormones \& Behavior, 12, 112-163.

BECKER, J. B., \& BEER, M. E. (1986). The influence of estrogen on nigrostriatal dopamine activity: Behavioral and neurochemical evidence for both pre- and post-synaptic components. Behavioral Brain Research, 19, 27-33.

Bedard, P. J., Boucher, R., Daigle, M., Di Paolo, T. (1985). Physiological doses of estradiol can increase lingual dyskinesia and cerebrospinal fluid homovanilic acid in monkeys. Neuroscience Letters, 58, 327-331

Chiodo, L. A., \& CAggiUla, A. R. (1983). Substantia nigra dopamine neurons: Alterations in basal discharge rates and autoreceptor sensitivity induced by estrogen. Neuropharmacology, 22, 593-599.

Chiodo, L. A., Caggiula, A. R., \& Saller, C. F. (1981). Estrogen potentiates the stereotypy induced by dopamine agonists in the rat. Life Sciences, 28, 827-835

Di Paolo, T., Rouillard, C., \& Bedard, P. (1985). $17 \beta$-estradiol at a physiological dose acutely increases dopamine turnover in rat brain. European Journal of Pharmacology, 117, 197-203.

Fink, J. S., \& Sith, G. P. (1980a). Mesolimbic and mesocortical dopaminergic neurons are necessary for normal exploratory behavior in rats. Neuroscience Letters, 17, 61-65. 
FINk, J. S., \& Smith, G. P. (1980b). Mesolimbicocortical dopamine terminal fields are necessary for normal locomotor and investigatory exploration by rats. Brain Research, 199, 359-384.

GANDelman, R. (1983). Gonadal hormones and sensory function. Neuroscience \& Biobehavioral Reviews, 7, 1-17.

GoRDON, J. H. (1980). Modulation of apomorphine-induced stereotypy by estrogen: Time course and dose response. Brain Research Bulletin, 5, 679-682.

HAskTns, J. T., Moss, R. L. (1983). Action of estrogen and mechanical vaginocervical stimulation on the membrane excitability of hypothalamic and midbrain neurons. Brain Research Bulletin, 10, 489-496.

Joyce, E. M., Stinus, L., Iversen, S. D. (1983). Effect of injection of 6-OHDA into either nucleus accumbens septi or frontal cortex on spontaneous and drug-induced activity. Neuropharmacology, 22, 1141-1145.

Joyce, J. N., Montero, E., \& Van Hartesveldt, C. (1984). Dopamine-mediated behaviors: Characteristics of modulation by estrogen. Pharmacology, Biochemistry \& Behavior, 21, 791-800.

JoyCe, J. N., VAN HARTESVeldT, C. (1984). Estradiol application to one striatum produces postural deviation to systemic apomorphine. Pharmacology, Biochemistry \& Behavior, 20, 575-581.

Kant, G. J., Mougey, E. H., Meyerhoff, J. L. (1986). Diurnal variation in neuroendorocrine response to stress in rats: Plasma ACTH, $\beta$-endorphin, $\beta$-LPH, corticosterone, prolactin and pituitary cyclic AMP responses. Neuroendocrinology, 43, 383-390.

Nabekura, J., OOmura, Y., Minami, T., Mizuno, Y., \&ukuda, A. (1986). Mechanism of the rapid effect of $17 \beta$-estradiol on medial amygdala neurons. Science, 233, 226-228.

VAN HartesveldT, C., \& Joyce, J. N. (1986). Effects of estrogen on the basal ganglia. Neuroscience \& Biobehavioral Reviews, 10, 1-14.

VAughn, D. M., VIIK, K., C CoOPER, B. R. (1985). The novel antipsychotic BW 234U antagonizes environmentally induced elevation of dopamine metabolism in prefrontal cortex. Society for Neuroscience Abstracts, 11, 115.

WeST, C. H. K., Michael, R. P. (1985). Responses in dopamine terminal regions to olfactory and somatosensory stimulation in rats. Society for Neuroscience Abstracts, 11, 207.

West, C. H. K., Michael, R. P. (1986). Time-dependent modulation by estrogen of amphetamine-induced hyperactivity in male rats. Pharmacology, Biochemistry \& Behavior, 25, 919-923.

(Manuscript received February 3, 1988; revision accepted for publication May 23, 1988.) 